\title{
N-3 polyunsaturated fatty acids reduces post-operative atrial fibrillation incidence in patients undergoing "on-pump" coronary artery bypass graft surgery
}

\author{
I N-3 PUFA riducono l'incidenza di fibrillazione atriale \\ post-operatoria nei pazienti sottoposti alla rivascolarizzazione \\ chirurgica in circolazione extracorporea
}

\author{
Mario Sorice1, Francesco Paolo Tritto1, Chiara Sordelli2, Rosario Gregorio1, Luigi Piazza1
}

\begin{abstract}
N$-3 polyunsaturated fatty acids reduces postoperative atrial fibrillation incidence in patients undergoing "on-pump" coronary artery bypass graft surgery. M. Sorice, F.P. Tritto, C. Sordelli, R. Gregorio, L. Piazza.

Background: The aim of this study was to evaluate the efficacy of preoperative and postoperative therapy with n-3 polyunsaturated fatty acids in reducing the incidence of atrial fibrillation after coronary artery bypass graft surgery.

Methods: 201 patients undergoing coronary artery bypass graft surgery were randomized to 1) a control group (105 patients), or 2 ) n-3 polyunsaturated fatty acids $2 \mathrm{~g} /$ day group ( 96 patients) for at least 5 days before surgery and until hospital discharge. Groups were further subdivided in four subgroups according to the operative technique: "off-pump" or "on-pump". The primary end point was to evaluate the reduced incidence of postoperative in-hospital atrial fibrillation in the (N-3 PUFA) group. Secondary end points were the impact of the surgical technique on the incidence of postoperative arrhythmia and the impact of n-3 polyunsaturated fatty acids therapy on post-operative hospital length of stay.
\end{abstract}

Results: The overall incidence of post-operative atrial fibrillation was $17.4 \%(35 / 201)$. The arrhythmia occurred in $11.4 \%(11 / 96)$ of the patients in therapy with $n-3$ polyunsaturated fatty acids and in $22.8 \%(24 / 105)$ in the control groups. In particular, the statistical analysis of subgroups showed a significant reduction of postoperative atrial fibrillation only in the group including patients treated with $\mathbf{n - 3}$ polyunsaturated fatty acids undergoing "on-pump" coronary artery bypass graft surgery. The length of hospital stay did not differ among all groups.

Conclusions: N-3 polyunsaturated fatty acids administration significantly reduces the incidence of post-operative atrial fibrillation in patients undergoing "on-pump" coronary artery bypass graft surgery. $\mathrm{N}-3$ polyunsaturated fatty acids therapy is not associated with a shorter hospital stay.

Keywords: heart bypass, coronary artery bypass, offpump, cardiopulmonary bypass, fatty acids, omega-3, atrial fibrillation.

Monaldi Arch Chest Dis 2011; 76: 93-98.

\footnotetext{
1 Department of Cardiac Surgery, S. Anna e S. Sebastiano Hospital, Caserta, Italy; Via F. Palasciano, 81100 (CE), Italy.

2 Department of Cardiothoracic Sciences, Monaldi Hospital, Second University of Naples, Naples; Via L. Bianchi, 80131 (NA), Italy.
}

Corresponding author: Francesco Paolo Tritto; Via Vetriera, 12; I-80132 Napoli, Italy; Phone number: 081407750; Mobile phone number: 3498753704

\section{Introduction}

Atrial fibrillation (AF) is a frequent complication following coronary artery bypass graft (CABG) surgery (11-40\%) [1-2], usually occurring on the second or third post-operative day. Different studies demonstrated that post-operative AF can lead to discomfort and hemodynamic instability with an increased hospital length stay [3]. Several drugs are used for prevention of post-operative AF, such as beta-blockers, ACE inhibitors and statins. However during the last few years the attention on $\mathrm{n}-3$ polyunsaturated fatty acids (PUFA) has increased: previous studies demonstrated anti-arrhythmic properties reducing the risk of sudden cardiac death and ventricular arrhythmias [4].

Other studies found that the human consumption of fish induced- high plasma PUFA concentration is was associated with a lower incidence of AF in a 12year follow-up study [5]. A recent trial investigated the efficacy of pre-operative and post-operative treatment with PUFA in preventing the occurrence of AF after coronary artery bypass graft surgery; the investigators found that fish oil supplementation for at least 5 days until surgery and continued after surgery was able to reduce the incidence of postoperative $\mathrm{AF}$ resulting in a shorter hospital stay [6]. Other authors recently showed that preoperative PUFA therapy was associated with a decreased incidence of early, but not late, AF after cardiac surgery [7].

The causes and pathogenesis of this complication are multi-factorial; recently, inflammatory mechanisms have also been involved [8]. It was recently reported that in sterile pericarditis model AF is associated with inflammation and n-3 PUFA 
could have a role in reducing the production of proinflammatory cytokines [9].

In the present study we hypothesized that preoperative and post-operative treatment with n-3 PUFA can prevent the occurrence of $\mathrm{AF}$ after CABG, taking into account the different surgical technique ("on-pump" or "off-pump" CABG).

\section{Methods}

\section{Study population}

From September 2009 to October 2010, 273 consecutive patients underwent CABG in our Institution. Among these, 72 were excluded. Exclusion criteria were: previous history of AF (12 pts), previous anti-arrhythmic treatment (unless with betablockers, Ca-antagonists)(10 pts), severe valvular disease requiring surgery (32 pts), different technical strategy of the surgeon (18 pts). Inclusion criteria were: $>18$ years, normal sinus rhythm and stable hemodynamic condition before cardiac surgery.

Two hundred and one consecutive eligible patients (164 men and 37 women; mean age $63 \pm 10$ years) undergoing CABG were enrolled in our study. All enrolled patients provided written informed consent to take part in the study.

\section{Study design}

These 201 eligible patients were assigned by randomization to a control group and to the PUFA (commercially available by Sigma Tau, Industrie Farmaceutiche Riunite, s.p.a. Italy) group. According to the surgical technique adopted, the groups were further divided into subgroups as follow: G1 group for PUFA patients operated with "off-pump" technique; G2 group for PUFA patients operated with "on-pump" technique; G3 group for patients operated with "off-pump" technique and not taking PUFA; G4 group for patients operated with "onpump" technique and not taking PUFA.

The choice of operating technique was randomized. The groups and the subgroups were selected according to homogeneity criteria.

This was a randomized prospective controlled study with parallel groups; the protocol was approved by the ethics committee of our institution.

Among the 201 patients enrolled, 96 were treated with PUFA-n3: they were administered with a daily dose of two gelatinous capsules containing 850 to $882 \mathrm{mg}$ eicosapentaenoic acid and docosahexaenoic acid as ethyl esters in the average ratio of EPA/DHA 1:2 beginning five days before surgery and going on until discharge. The remaining $105 \mathrm{pa}-$ tients constituted the control group. The surgical access was obtained with a median sternotomy in all patients. Induction and anesthesia was the same in all patients. Myocardial protection was afforded with normotermic hematic potassium cardioplegia. Calculated heparin dosages were different in the two techniques (halved values in the off-pump patients). All anastomoses were sutured by hand.

Intensive care unit (ICU) standardized protocol implied 48 hours stay after surgery. Once discharged, patients were transferred to a monitored unit, where 3-lead telemetric monitoring was performed continuously for at least 4 days after the op- eration. In addition, patients had a 12-lead ECG daily until hospital discharge and an ECG was also performed in case of symptoms or when an arrhythmia was suspected. Patients who had AF were treated with intravenous injection of amiodarone and discharged with instructions to undergo oral amiodarone therapy for at least 30 days. Demographic and clinical pre-operative data are summarized in Table 1.

\section{Study endpoints}

The primary end point of the study was to evaluate the incidence of post-operative in-hospital $\mathrm{AF}$ in the four groups. AF was defined as an episode of $\mathrm{AF}$ that lasted $>5$ minutes, or any episode requiring a treatment for hemodynamic compromise. After an episode of AF or at hospital discharge, formal study participation ended. Secondary end points were:

1) impact of surgical technique ("off-pump"/ "onpump") on the incidence of post-operative AF; 2) length of post-operative hospital stay.

\section{Statistical analysis}

The occurrence of post-operative AF in the two treatment groups was tested with the odds ratio (OR) of two-binomial proportion analysis. Mean $( \pm \mathrm{SD})$ were calculated for continuous variables, and frequencies were measured for categorical variables. Differences between groups were analyzed by an unpaired Student $\mathrm{T}$ test for continuous variables, or chi-square tests in the case of categorical variables. A $p$ value $<0.05$ was considered significant.

\section{Results}

105 patients were assigned by randomization to the control group and 96 to the PUFA group. G1 group comprised 45 of 201 patients $(22.4 \%) ; \mathrm{G} 2$ group comprised 51 patients $(25.4 \%)$; G3 group comprised 48 patients (23.9\%); G4 group comprised 57 patients $(28.3 \%)$ (Table 2 ).

Implanted grafts were left internal mammary artery (LIMA) in 200 pts, right internal mammary artery (RIMA) in 25 pts, radial artery (RA) in 33 pts and sapheneous vein (SVG) in 165 pts.

The overall incidence of post-operative $\mathrm{AF}$ was $17.4 \%$ (35 of 201 patients). Post-operative AF occurred in $11.4 \%$ of the patients (11 of 96 patients) in PUFA therapy (G1-G2) and in $22.8 \%$ (24 of 105) of the control groups $(\mathrm{G} 3-\mathrm{G} 4)(\mathrm{OR} 0.43$; $95 \%$ CI $0.2-$ $0.95 ; \mathrm{p}=0.033$ by chi-square). In particular in the various subgroups the incidence of $\mathrm{AF}$ was: $11.1 \%$ in G1 (5 of 45), $11.7 \%$ in G2 (6 of 51), $12.5 \%$ in G3 ( 6 of 48) and of $31.6 \%$ in G4 (18 of 57). The statistical analysis showed a significant effect in favour of the PUFA group compared with controls only between G2 and G4 groups (OR 0.28; p $=0.013$ by chi-square) (Figure 1). No differences were found between $\mathrm{G} 1$ and $\mathrm{G} 3$ groups (OR 0.87; $\mathrm{p}=0.835$ by chi-square).

No differences were found among groups regarding length of hospital stay ( $8 \pm 4$ days, $\mathrm{p}=\mathrm{ns}$ ).

No patient developed allergic skin rash during PUFA therapy. Non fatal postoperative complications occurred in 10 patients $(10.7 \%)$ in which "offpump" technique was adopted (G1 and G3 groups): 
four kidney failure, four implantation of intra-aortic pumping balloon, two respiratory failure; neigher in 12 patients $(11.1 \%)$ in which "on-pump" technique was performed (G2 and G4 groups): three kidney failure, four implantation of intra-aortic pumping balloon, two respiratory failure, two mediastinitis, one surgical wound infection. Surgical re-exploration for bleeding was required in three patients operated with off-pump technique. There was no operative mortality.

Table 1. - Baseline clinical characteristics

\begin{tabular}{|c|c|c|c|c|c|c|}
\hline & G1 $(n=45)$ & G2 $(n=51)$ & G3 $(n=48)$ & G4 $(n=57)$ & pG1-G3 & pG2-G4 \\
\hline Age (yrs) & $64 \pm 10$ & $63 \pm 10$ & $63 \pm 10$ & $63 \pm 9$ & 0.65 & 0.97 \\
\hline Male gender & $37(82 \%)$ & $39(76 \%)$ & $42(87 \%)$ & $46(80 \%)$ & 0.70 & 0.79 \\
\hline Diabetes mellitus & $17(38 \%)$ & $23(45 \%)$ & $20(42 \%)$ & $25(44 \%)$ & 0.85 & 0.93 \\
\hline Systemic Hypertension & $36(80 \%)$ & $33(65 \%)$ & $26(54 \%)$ & $34(60 \%)$ & 0.01 & 0.73 \\
\hline Dyslipidemia & $16(36 \%)$ & $18(35 \%)$ & $27(56 \%)$ & $24(42 \%)$ & 0.08 & 0.58 \\
\hline COPD & $12(27 \%)$ & $15(29 \%)$ & $19(40 \%)$ & $21(37 \%)$ & 0.27 & 0.50 \\
\hline Renal failure & $5(11 \%)$ & $3(6 \%)$ & $2(4 \%)$ & $1(2 \%)$ & 0.37 & 0.56 \\
\hline NYHA & $1.7 \pm 0.5$ & $1.8 \pm 0.6$ & $1.7 \pm 0.5$ & $1.9 \pm 0.6$ & 0.72 & 0.58 \\
\hline \multicolumn{7}{|l|}{ Medical therapy: } \\
\hline Calcium channels blockers & $7(16 \%)$ & $12(24 \%)$ & $4(8 \%)$ & $9(16 \%)$ & 0.38 & 0.42 \\
\hline$\beta$-blockers & $22(49 \%)$ & $29(57 \%)$ & $33(69 \%)$ & $37(65 \%)$ & 0.08 & 0.51 \\
\hline ACE-inhibitors & $24(53 \%)$ & $34(67 \%)$ & $24(50 \%)$ & $29(51 \%)$ & 0.93 & 0.13 \\
\hline Statins & $28(62 \%)$ & $30(59 \%)$ & $30(62 \%)$ & $44(77 \%)$ & 0.83 & 0.07 \\
\hline Acetilsalicilic acid & $27(60 \%)$ & $33(65 \%)$ & $25(52 \%)$ & $38(67 \%)$ & 0.57 & 0.98 \\
\hline Nitrates & $16(36 \%)$ & $23(45 \%)$ & $20(42 \%)$ & $25(44 \%)$ & 0.7 & 0.92 \\
\hline Diuretics & $10(22 \%)$ & $8(16 \%)$ & $1(2 \%)$ & $8(14 \%)$ & 0.01 & 0.98 \\
\hline LV ejection fraction $(\%)$ & $53 \pm 10$ & $51 \pm 10$ & $53 \pm 8$ & $53 \pm 7$ & 0.87 & 0.19 \\
\hline Left atrium AP dimension (mm) & $41 \pm 6$ & $42 \pm 6$ & $42 \pm 8$ & $38 \pm 4$ & 0.56 & 0.02 \\
\hline
\end{tabular}

G1 = Off-pump CABG, treated with PUFA. G2 = On-pump CABG, treated with PUFA. G3 = Off-pump CABG without PUFA. G4 = On-pump CABG without PUFA. NYHA = New York Heart Association. COPD = chronic obstructive pulmonary. PUFA $=$ polyunsaturated fatty acids. $\mathrm{ACE}=$ angiotensin-converting enzyme. $\mathrm{LV}=$ left ventricle. $\mathrm{AP}=$ antero-posterior.

Table 2. - Results.

\begin{tabular}{lcccccc}
\hline G1 (n=45) & G2 $(\mathbf{n}=\mathbf{5 1})$ & $\mathbf{G 3}(\mathbf{n = 4 8})$ & $\mathbf{G 4}(\mathbf{n = 5 7})$ & pG1-G3 & pG2-G4 \\
\hline ATRIAL FIBRILLATION INCIDENCE & $5(11.1 \%)$ & $6(11.7 \%)$ & $6(12.5 \%)$ & $18(31.6 \%)$ & 0.835 & 0.013 \\
\hline TOTAL GRAFTS & 90 & 110 & 103 & 120 & 0.65 & 0.87 \\
\hline Left internal mammary artery grafts & $44(97.7 \%)$ & $51(100 \%)$ & $48(100 \%)$ & $57(100 \%)$ & 0.67 & 0.13 \\
\hline Right internal mammary artery grafts & $5(11.1 \%)$ & $5(9.8 \%)$ & $11(22.9 \%)$ & $4(7 \%)$ & 0.7 & 0.9 \\
\hline Radial artery grafts & $8(17.7 \%)$ & $6(11.7 \%)$ & $12(25 \%)$ & $7(12.3 \%)$ & 0.43 & 0.85 \\
\hline Sapheneous vein grafts & $33(73.3 \%)$ & $48(94.1 \%)$ & $32(66.6 \%)$ & $52(91.2 \%)$ & 0.18 & 0.34 \\
\hline AORTIC CROSS-CLAMP (mean minutes) & & 55.3 & & 57.4 & & 0.59 \\
\hline CEC (mean minutes) & & 80.9 & & 81.2 & & 0.95 \\
\hline LENGTH OF HOSPITAL STAY (days) & $8.2 \pm 2.5$ & $8.6 \pm 3.8$ & $8.3 \pm 3.1$ & $8.8 \pm 4.9$ & 0.79 & 0.75 \\
\hline
\end{tabular}

G1 = Off-pump CABG, treated with PUFA. G2 = On-pump CABG, treated with PUFA. G3 = Off-pump CABG without PUFA. $\mathrm{G} 4=$ On-pump CABG without PUFA. PUFA = polyunsaturated fatty acids. $\mathrm{CEC}=$ extracorporeal circulation. 


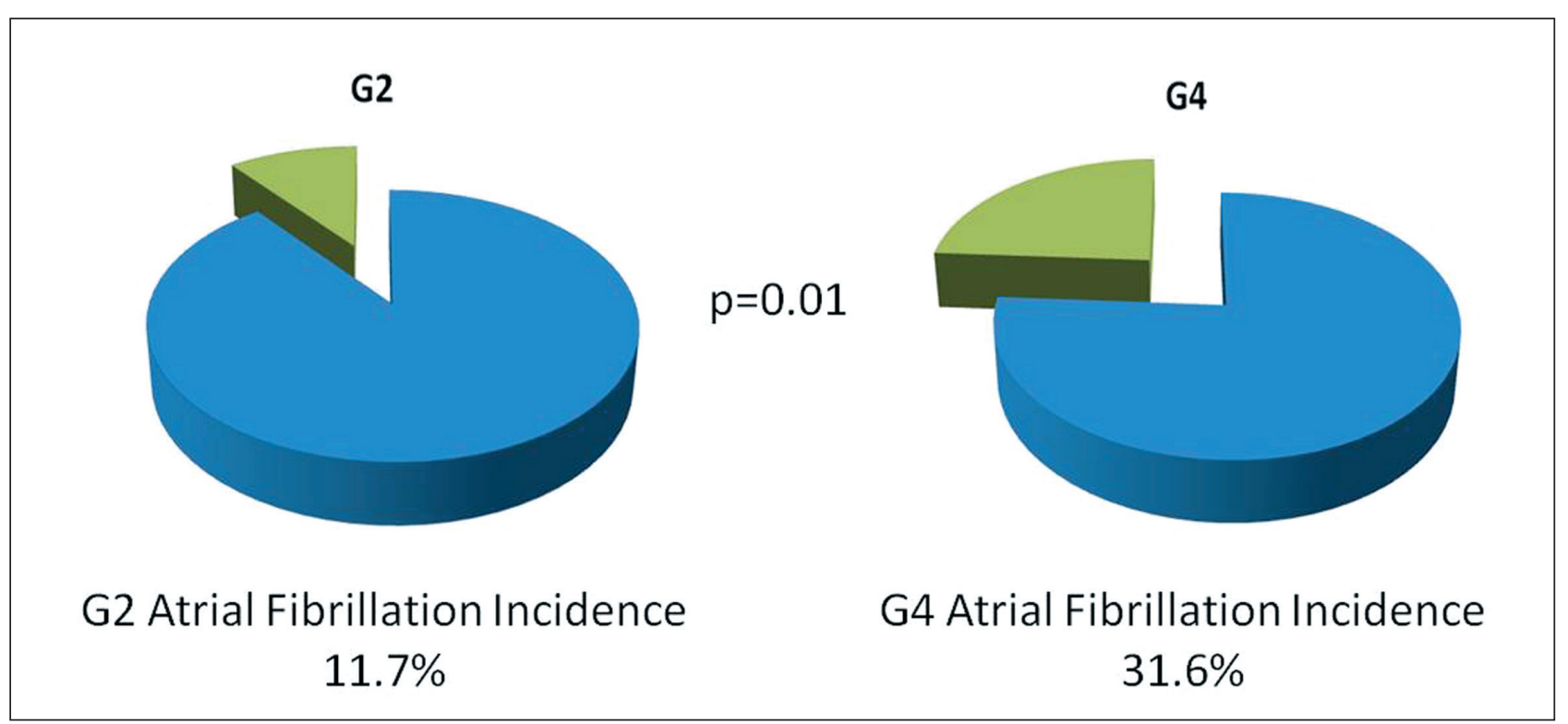

Figure 1. - G2=On-pump CABG, treated with PUFA. G4=On-pump CABG without PUFA. PUFA= polyunsaturated fatty acids.

\section{Discussion}

$\mathrm{AF}$ is the most common complication associated with $\mathrm{CABG}$ [1-2] and can lead to discomfort and hemodynamic instability [3]. During the last few years the attention on PUFAs is increased because of their documented beneficial effects in preventing the occurrence of post-operative AF. The first demonstration of the beneficial effect of PUFA derives from the observation that consumption of fish or fish oil reduces the incidence of fatal ventricular arrhythmias, manifested as coronary death or sudden death. It is biologically plausible that this observed relationship is due to effects of longchain n-3 fatty acids in fatty fish [10]. Anti-arrhythmic action of PUFAs can be attributed to their inhibiting capacity of some ion channels and in particular sodium current, calcium current and potassium currents [11]. These effects cause an increased electrical stability that may result in a protection against $\mathrm{AF}$ [12]. In cultured rat atrial myocytes, n-3 fatty acids reduce induced asynchronous contractile activity [13], suggesting that $n-3$ fatty acids from fish may have anti-arrhythmic effects on atrial muscle. Another study has demonstrated that oral n-3 PUFAs supplementation, in an animal model of atrial remodelling and $\mathrm{AF}$, reduced $\mathrm{AF}$ inducibility and maintenance by activating matrix metalloproteinase-9 mRNA expression and attenuating collagen turnover [14]. However, pathophysiological mechanisms of postoperative AF were the focus of many studies and still remain partly unknown. Scientists are increasingly supporting the hypothesis of an important role of surgery-related inflammatory cascade as the leading cause of post-operative AF. Many studies evaluated CRP levels as prototypic marker of inflammation. Bruins et al. found that on the second post-operative day CRP levels peak as the incidence of AF [15]. Another study showed that CRP is not only associated with the presence of $\mathrm{AF}$ but it is also a risk factor for this specific arrhythmia [16]. A recent study found that dietary n-
3 PUFA supplementation attenuates the inducibility and maintenance of $\mathrm{AF}$ in a canine sterile pericarditis model by reducing the production of proinflammatory cytokines such as CRP, IL-6, TNF- $\alpha$ [9]. There are several clinical studies reporting that PUFAs have beneficial effects in inflammatory disease [17-18]. The anti- inflammatory effects of PUFA are due to various mechanisms: decreased proportions of arachidonic acid and eicosanoids in inflammatory cells [19], decrease in chemotaxis of human neutrophils and monocytes [19], reduced expression of adhesion molecules on monocytes, macrophages and endothelial cells surface [20], reduced synthesis of inflammatory cytokines [9]. Consequently we can hypothesize that an anti-inflammatory action of PUFAs could play an important role in the prevention of $\mathrm{AF}$ after $\mathrm{CABG}$.

In this trial we have demonstrated that PUFA administration significantly reduces the overall incidence of postoperative $\mathrm{AF}$ in patients undergoing CABG (11.4\% absolute risk reduction, $49.8 \%$ relative risk reduction). In particular a statistically significant reduction of AF post-operative incidence was observed only in patients undergoing "onpump" CABG (19.8\% absolute risk reduction, $62.7 \%$ relative risk reduction). This finding may further support the hypothesis of an anti-inflammatory PUFA action considering that "on-pump" CABG induces a systemic inflammatory response by triggering the production and release of inflammatory mediators. Several studies documented the efficacy of PUFA administration in preventing AF after coronary artery bypass graft surgery. Calò et al. showed that the use of PUFAs during hospitalization in patients undergoing $\mathrm{CABG}$ significantly reduced the incidence of postoperative AF and was associated with a shorter hospital stay, reporting the first direct evidence of an atrial anti-arrhythmic effect of PUFAs [6]. Other authors recently showed that pre-operative PUFA therapy was associated with a decreased incidence of early, but not late, AF after cardiac surgery [7]. A recent study did not confirm 
these previous findings, supporting the hypothesis that omega 3-PUFAs do not reduce the risk of AF after coronary artery bypass graft surgery; however, these results could be hampered by limited study population [21]. Our study is in agreement with previous studies reporting a beneficial effect of PUFA therapy in reducing the incidence of post-operative $\mathrm{AF}$; furthermore, we demonstrated the PUFA beneficial effect is strictly dependent on specific surgical technique with benefit evident in patients undergoing "on-pump" CABG.

\section{Conclusion}

Pre-operative PUFA therapy in patients undergoing "off-pump" CABG has no substantial benefits. N-3 polyunsaturated fatty acids administration, despite not being able to reduce hospital stay, significantly reduces the incidence of post-operative atrial fibrillation in patients undergoing coronary artery bypass graft with "on-pump" technique.

\section{Riassunto}

Background: La fibrillazione atriale (FA) è una complicanza che si osserva di frequente nella chirurgia della rivascolarizzazione miocardica $(B P A C)$ e può comportare un incremento dei tempi di degenza ospedaliera. Numerosi studi hanno documentato che gli $n-3$ acidi grassi poliinsaturi (N-3 PUFA) riducono l'incidenza di FA post-operatoria.

Obiettivi: Scopo di questo studio è di valutare l'efficacia del trattamento preoperatorio e postoperatorio con PUFA al fine di ridurre l' incidenza di FA nei pazienti sottoposti a rivascolarizzazione chirurgica, valutando anche le differenze tecniche operatorie adottate ("on-pump" o "off-pump").

Metodi: Per un periodo di circa un anno, 201 pazienti sottoposti a BPAC sono stati randomizzati in 1) gruppo di controllo (105 pazienti), o 2) gruppo PUFA: 2 g/die (96 pazienti) per almeno 5 giorni prima della chirurgia e fino alla dimissione ospedaliera. I gruppi sono stati ulteriormente divisi in 4 sottogruppi (G1-G4) a seconda della tecnica operatoria utilizzata: "off-pump" o "on-pump". L'end point primario dello studio è stato di valutare l' incidenza della FA post-operatoria ospedaliera nei diversi gruppi. End point secondario è stato quello di valutare l'impatto della tecnica chirurgica ("offpump”/ “on-pump”) sull' incidenza della FA postoperatoria e sul tempo di degenza ospedaliera.

Risultati: L'incidenza post-operatoria di FA è stata del 17.4\% (35 pazienti su 201). L'aritmia si è verificata nell' $11.4 \%$ (11/96) dei pazienti in terapia con PUFA e nel $22.8 \%$ (24/105) nel gruppo di controllo. In particolare l'analisi statistica dei sottogruppi ha mostrato una riduzione significativa di FA post-operatoria solo nel gruppo dei pazienti trattati con PUFA ed operati con tecnica “on-pump”. La lunghezza della degenza ospedaliera non differiva tra $i$ vari sottogruppi.

Conclusione: La somministrazione di PUFA riduce in maniera significativa l'incidenza postoperatoria di FA nei pazienti sottoposti a BPAC con tecnica "on pump". La terapia con PUFA non è associata a riduzione della degenza ospedaliera.

\section{ABBREVIATIONS}

Atrial fibrillation (AF)

Coronary artery bypass graft $(\mathrm{CABG})$

N-3 polyunsaturated fatty acids (PUFA)

Intensive care unit (ICU)

Odds ratio (OR)

Left internal mammary artery (LIMA)

Right internal mammary artery (RIMA)

Radial artery (RA)

Sapheneous vein (SVG)

\section{References}

1. Leitch JW, Thomson D, Baird DK, Harris PJ. The importance of age as a predictor of atrial fibrillation and flutter after coronary artery bypass grafting. $J$ Thorac Cardiovasc Surg 1990; 100: 338-342.

2. Creswell LL, Schuessler RB, Rosenbloom M, Cox JL. Hazards of postoperative atrial arrhythmias. Ann Thorac Surg 1993; 56: 539-549.

3. Ommen SR, Odell JA, Stanton MS. Atrial arrhythmias after cardiothoracic surgery. $N$ Engl J Med 1997; 336: 1429-1434.

4. Jouven X, Charles M-A, Desnos M, Ducimetiere P. Circulating nonesterified fatty acid level as a predictive risk factor for sudden death in the population. Circulation 2001; 104: 756-61.

5. Mozaffarian D, Psaty BM, Rimm EB, et al. Fish intake and risk of incident atrial fibrillation. Circulation 2004; 110: 368- 373.

6. Calò L, Bianconi L, Colivicchi F, et al. N3-Fatty Acids for the prevention of Atrial Fibrillation After Coronary Artery Bypass Surgery: a randomized, controlled trial. $J$ Am Coll Cardiology 2005; 45 (10): 1723-8.

7. Mariscalco G, Sarzi Braga S, Banach M, et al. Preoperative $n-3$ polyunsaturated fatty acids are associated with a decrease in the incidence of early atrial fibrillation following cardiac surgery. Angiology 2010; 61 (7): 643-50.

8. Christopher J. Boos, Richard A. Anderson and Gregory Y.H. Lip Is atrial fibrillation an inflammatory disorder? Eur Heart J 2006; 27(2): 136-149.

9. Zhong Zhang, Chao Zhang, Hongtao Wang, et al. N-3 polyunsatured fatty acids prevents atrial fibrillation by inhibiting inflammation in a canine sterile pericarditis model. Int J of Cardiol 2010 Sep 8, in press.

10. Mozaffarian D, Rimm EB. Fish intake, contaminants, and human health: evaluating the risks and the benefits. JAMA 2006; 296: 1885-99.

11. Leaf A. The electrophysiological basis for the antiarrhythmic action of polyunsaturated fatty acids. Eur Heart $J$ 2001; 3 Suppl D: D98-105.

12. McLennan PL, Bridle TM, Abeywardena MY, Charnock JS. Dietary lipid modulation of ventricular fibrillation threshold in the marmoset monkey. Am Heart J 1992; 123: 1555-61.

13. Jahangiri A, Leifert WR, Patten GS, McMurchie EJ. Termination of asynchronous contractile activity in rat atrial myocytes by n-3 polyunsaturated fatty acids. $\mathrm{Mol}$ Cell Biochem 2000; 206: 33-41.

14. Laurent G, Moe G, Hu X, et al. Long chain n-3 polyunsaturated fatty acids reduce atrial vulnerability in a novel canine pacing model. Cardiovasc Res 2008; 77: 89-97.

15. Bruins P, te Velthuis H, Yazdanbakhsh AP, et al. Activation of the complement system during and after cardiopulmonary bypass surgery: postsurgery activation involves C-reactive protein and is associated with postoperative arrhythmia. Circulation 1997; 96(10): 3542-8.

16. Aviles RJ, Martin DO, Apperson-Hansen C, et al. Inflammation as risk factor for atrial fibrillation. Circulation 2003; 108(24): 3006-3010. 
17. James MJ, Gibson RA, Cleland LG. Dietary polyunsaturated fatty acids and inflammatory mediator production. Am J Clin Nutr 2000; 71: 343S-348S.

18. Mori TA, Chem CP, Beilin LJ. Omega-3 fatty acids and inflammation. Curr Atheroscler Rep 2004; 6: 461-7.

19. Lee TH, Hoover RL, Williams JD, et al. Effect of dietary enrichment with eicosapentaenoic and docosahexaeonic acids on in vitro neutrophil and monocyte leukotriene generation and neutrophil function. N Engl J Med 1985; 312(19): 1217-24.
20. Miles EA, Wallace FA, Calder PC. Dietary fish oil reduces intercellular adhesion molecule and scavenger receptor expression on murine macrophages. Atherosclerosis 2000; 152(1): 43-50.

21. Saravanan P, Bridgewater B, West AL, O'Neill SC, Calder PC, Davidson NC. Omega-3 fatty acid supplementation does not reduce risk of atrial fibrillation after coronary artery bypass surgery: a randomized, double blind, placebo-controlled clinical trial. Circ Arrhythm Electrophysiol 2010; 3(1): 46-53. 